\title{
Research on Platform Pricing Mode Considering Users' Psychological Cost
}

\author{
Liu Dawei, Yao Min, Li Qian* \\ College of Big Data Application and Economic, GuiZhou University of Finance and Economics, Guiyang, \\ China
}

Keywords: platform, pricing mode, psychological cost

\begin{abstract}
After the initial free phase, platform will try to charge some or all users to compensate costs or increase profitability. In order to analyse the influence of the psychological cost on the choice of the charging method for the platforms, a basic model is constructed. Then the selection of the supplier is analyzed through static game and the dynamic game under the monopoly and duopoly respectively. For monopolists, if only one charging method can be choose, the usage charges will be choose when the psychological cost is low, else it will choose membership charges. If the supplier can choose both methods simultaneously, the option to provide two charging methods at the same time will obtain higher profits. In a duopoly, one firm uses membership charges and the other uses usage charges whether it is static game or dynamic game.
\end{abstract}

\section{Introduction}

After the platform has successfully achieved the goal of "getting both parties to the deck" after the initial free phase, it will try to charge some or all users to compensate costs or increase profitability. For example, in 2017, Tencent's social network revenue reached 8 billion dollars, an increase of 52\% over the previous year; Jingdong Mall's Plus members in September 2018 had exceeded 10 million, becoming the largest paid member group in China's e-commerce industry; Netflix already has more than 130 million paid members worldwide. Faced with users who are accustomed to various products such as free chat, shopping, and audio-visual content on the Internet, the platform's choice of pricing mode directly affects its own survival and development space.

There are two general pricing modes of the platform. These modes are membership fee, where up-front payment allows unrestricted use, and transaction fee, where payments are tailored to use. How the platform chooses between the two pricing modes depend on the user's response to different pricing modes. Different from the membership fee mode, each time the user pays an additional psychological cost in the transaction fee mode. How the psychological cost affects the selection of the platform's pricing mode is concerned by platform operators.

The initial focus on the platform market stems from its special pricing mode. Rochet and Tirole (2003) proposed the concept of a two-sided market and defined it in terms of price structure; further research also specifically discussed the different effects of membership fee and transaction fee ${ }^{[1]}$. The main purpose of the two-sided market theory's attention to the price structure is to explain the mechanism of the inclining price structure in markets with cross-network externalities. Armstrong (2006) indicate that due to the existence of cross-network externalities, platform often develop lower price (sometimes free or even negative price) in order to allow that side to accumulate as many users as possible, and to improve the utility of users on the other side through cross-network externalities, so that higher prices can be set on the other side to obtain profits ${ }^{[2]}$. Initially, the platform can only choose a unified tilted price structure under the assumption of no difference users. In reality, users are obviously more complicated, and the original subsidy can also become the platform's profit source. Some researchers have noticed the impact of pricing modes on the benefits of all parties. Chen Peng, Xiong Li, and Yang Lu (2018) used computer simulation technology to study the changes in user utility when digital content platforms adopt a membership pricing model, a transaction pricing model, and a combination, indicating that mixed pricing modes are more in line with users profit maximization ${ }^{[3]}$. Xia Dejian and Wang Yong (2018) specialize in pricing modes based on transaction fee, further distinguishing three different types of fee according to 
transaction volume, transaction amount, and number of transactions, and discuss the impact of their combination on prices and the profits of all parties ${ }^{[4]}$. Liu Weiqi and Zhang Su (2017) set a case where there is only a membership fee and no transaction fee when studying the pricing issue of platform mergers ${ }^{[5]}$.

The research on platform pricing mainly focuses on the level of charging, and rarely pays attention to the pricing mode. As the level of psychological cost changes, the user's tendency to choose between the two pricing modes will change, and the pricing mode of the product provider will also change accordingly. The theory of psychological accounts is an important concept in behavioral economics. Thaler (1985) formally proposed the concept of psychological accounts ${ }^{[6]}$, and Prelec and Loewenstein (1998) proposed a dual-channel psychological account model, describing the interaction between "the joy of consumption" and "the pain of payments" ${ }^{[7]}$. If the charge levels are the same, rational users will see no difference between the two mode. However, it is difficult to avoid the occurrence of psychological costs in search, bargaining, and supervision during the transaction process. And because users are bounded rationality, even if the cost of the users is exactly the same, since every transaction will have visible consumption, continuous consumption will incur a psychological cost in the psychological account. Soman (2001) confirmed that the continuously accumulated psychological cost will have a suppressive effect on consumption ${ }^{[8]}$. Shy (2008) shows that, because non-monetary costs occur at every transaction, users have tended to choose a fixed-rate membership fee mode despite the fact that the cost-per-view proves to be lower ${ }^{[9]}$. Dutta et al. (2013) also confirmed that non-monetary psychological accounts have a direct impact on corporate pricing ${ }^{[10]}$. Bi Wenjie, Chen Genyu and Chen Xiaohong (2015) studied the effect of psychological accounts on dynamic pricing and long-term profits of enterprises ${ }^{[11]}$. Li Aimei, Hao Mei et al. (2012) research on psychological accounts shows that when consumption and payment are closely linked, it will make people more apt to think about the pain of payment, which will reduce consumption pleasure ${ }^{[12]}$. The user's psychological cost mainly occurs in the mode of pay-per-use, and the psychological cost will have an important impact when determining the relative attractiveness of the two pricing modes.

In the research on platform pricing, the user's psychological cost has not been given due attention. The research focusing only on the price level ignores the impact of the pricing mode. There is no reasonable explanation can be found from existing research on how the platform chooses between the two pricing modes of selling and pay-per-use. This study introduces psychological cost into the pricing mode selection model, and analyzes the platform's pricing mode selection under the monopoly and duopoly market structure.

The rest of the paper is organized as follows. Section 2 proposes the model, section 3 describes the pricing mode selection of monopoly, and in the selection of duopoly was analyzed in section 4 , and we conclude the paper with Section 5.

\section{Model}

Assume that there are $\mathrm{N}$ users in the platform. The platform provides products to users through two methods: membership fee and transaction fee, with zero fixed cost. The usage frequency of user $i$ denoted as $\theta_{i}$, which follows a uniform distribution, that is, $\theta_{i} \sim \mathrm{U}\left[0, \theta_{H}\right]$, where $\theta_{H}$ represents the upper limit of the use frequency (especially, if $\theta_{H}=1, \theta_{i}$ can be used to indicate the probability that the user uses the product ); all users have the same utility $\phi$ per use. Users who use transaction fee incur a psychological cost $\mathrm{T}$ each time. In order to distinguish the parameters of the two charging modes in the model, the subscript $S$ is used to represent the relevant parameters of the membership fee mode, and the subscript $\mathrm{O}$ is used to represent the parameters of the transaction fee mode.

From the perspective of the user, only when $\phi-T \geq p_{O}$, user $i$ will use the transaction fee mode, which means that the net utility of each use $\phi_{i}-T$ must not be lower than the price paid for each use $p_{O}$; Similarly, the user will choose the membership fee mode only when $\theta_{i} \phi \geq p_{S}$. The expression to the left of the inequality indicates the cumulative utility obtained by the user. The 
surplus for user $i$ in the two charging modes respectively is:

Transaction fee

$$
U_{i o}\left(p_{O}\right)=\theta_{i}\left(\phi-p_{O}-T\right)
$$

Membership fee

$$
\mathrm{U}_{\mathrm{iS}}\left(\mathrm{p}_{\mathrm{S}}\right)=\theta_{\mathrm{i}} \phi-\mathrm{p}_{\mathrm{S}}
$$

When calculating the profits of the transaction fee mode, the payment takes place with each use. In contrast, the surplus is the utility subtract the membership fee in the membership fee mode. The monopoly and duopoly cases will be discussed separately later. Suppliers are sometimes only allowed to provide one charging mode. At this time, the supplier must choose between transaction fee and membership fee. If the supplier can provide two pricing modes at the same time, the selection of its charging mode is still affected by psychological costs. In the duopoly's competitive market structure, the selection of the supplier's charging model needs to take into account the psychological conditions as well as the information conditions and rules of the game.

\section{Pricing Mode for Monopolist}

If there is only one supplier in the market, it may need to choose one of the two pricing modes, or it may provide both pricing modes. Here we analyze the equilibrium price and profit firstly when the supplier can only choose one charging mode, and analyze the selection conditions for the monopolist, and then analyze the equilibrium and profit using both charging modes at the same time.

\subsection{Transaction fee}

If the monopolist uses the transaction fee mode separately, the buyer will choose to purchase the product only when the price of the transaction fee $p_{O} \leq \phi-T$. According to the assumption that the frequency of use is evenly distributed, the average frequency of use under the transaction fee mechanism is $\theta_{H} / 2$. The sales volume is $N \theta_{H} / 2$, and the platform's profit maximization model can be expressed as

$$
\max \quad N p_{O} \frac{\theta_{H}}{2}
$$

$$
\text { s.t. } p_{O} \leq \phi-T
$$

A Lagrangian function can be constructed and according to the Kuhn-Tucker condition, we can get

$$
\begin{gathered}
\frac{\partial F}{\partial p_{O}}=N \frac{\theta_{H}}{2}-\lambda=0 \\
\lambda\left[p_{O}-(\phi-T)\right]=0
\end{gathered}
$$

Since $\lambda \neq 0, \quad p_{O}-(\phi-T)=0$ 。 This means that for maximum profit, the monopolist will set the price per use as:

$$
p_{O}=\phi-T
$$

The profit of the transaction fee mode as

$$
\Pi_{O}\left(p_{O}\right)=N(\phi-T) \frac{\theta_{H}}{2}
$$

\subsection{Membership fee}

If the company provides products with membership fee, the number of members and profits of the monopolist are:

$$
\mathrm{M}_{S}\left(p_{S}\right)=\frac{N}{\theta_{H}}\left[\theta_{H}-\frac{p_{S}}{\phi}\right]
$$




$$
\Pi_{S}\left(p_{S}\right)=\mathrm{M}_{S}\left(p_{S}\right) p_{S}=\frac{N p_{S}}{\theta_{H}}\left[\theta_{H}-\frac{p_{S}}{\phi}\right]
$$

The monopoly platform membership fee is

$$
p_{S}=\frac{\theta_{H} \phi}{2}
$$

The number of members for maximize profit is $\frac{N}{2}$, and the maximum profit is:

$$
\Pi_{S}\left(p_{S}\right)=\frac{N \phi \theta_{H}}{4}
$$

\subsection{Using two pricing modes}

If the monopolist can use two charging modes at the same time, since there is no difference in products, users will only choose the one that is most beneficial to them: users who use more frequently will choose the membership fee, users who use less frequently will choose transaction fee. The conditions for users to choose membership fee instead of transaction fee can be expressed as: $U_{i S}\left(p_{S}\right)>U_{i O}\left(p_{O}\right)$, that is

$$
\theta_{i} \phi-p_{S}>\theta_{i}\left(\phi-p_{O}-T\right)
$$

For users who use it less frequently, the transaction fee set by the platform is still:

$$
p_{O}=\phi-T
$$

Substituting (13) into (12), users who use more frequently $\left(\theta>\frac{p_{S}}{\phi}\right)$ will choose the membership fee method, and users who use less frequently $\left(\theta<\frac{p_{S}}{\phi}\right)$ will choose transaction fee.

The total profit of the monopolist who provides both charging modes is:

$$
\Pi=\Pi_{S}+\Pi_{O}=\frac{N p_{S}}{\theta_{H}}\left(\theta_{H}-\frac{p_{S}}{\phi}\right)+\frac{N p_{O}}{2 \theta_{H}}\left(\frac{p_{S}}{\phi}\right)^{2}=\frac{N p_{S}}{\theta_{H}}\left(\theta_{H}-\frac{p_{S}}{\phi}+\frac{p_{O} p_{S}}{2 \phi^{2}}\right)
$$

According to the first-order conditions, the optimal price of the membership fee is:

$$
p_{S}=\frac{\theta_{H}^{2} \phi^{2}}{\phi+T}
$$

The market share of transaction fee and membership fee can be obtained:

$$
\begin{gathered}
\mathrm{MS}_{O}=\frac{\theta_{H} \phi}{\phi+T} \\
\mathrm{MS}_{S}=1-\frac{\theta_{H} \phi}{\phi+T}
\end{gathered}
$$

If two pricing modes can be used at the same time, the profit obtained by the monopolist is usually significantly higher than when only one charging model is provided. According to the results of market share (16) (17), we know that the monopolist will always provide the transaction fee charging model $\left(\mathrm{MS}_{O}>0\right)$; when the psychological cost continues to rise to $T>\phi\left(\theta_{H}-1\right)$, $\mathrm{MS}_{S}>0$, at this time, some users will choose to obtain the goods through membership fee.

\section{Pricing Mode for Duopolies}

The duopoly's game is mainly concerned with the choice charging mode. First consider the static games: each company can choose one of the two charging modes or use both charging modes at the same time, and the two companies make decisions at the same time, considering the game only play once. For user $i$, the net utility obtained of the two companies list in table 1 .

Table 1 Net utility obtained of the two companies.

\begin{tabular}{|c|c|c|}
\hline & Platform 1 & Platform 2 \\
\hline Transaction fee & $\mathrm{U}_{\mathrm{O} 1}\left(\mathrm{p}_{\mathrm{O} 1}\right)=\theta_{\mathrm{i}}\left(\phi-\mathrm{T}-\mathrm{p}_{\mathrm{O} 1}\right)$ & $\mathrm{U}_{\mathrm{O} 2}\left(\mathrm{p}_{\mathrm{O} 2}\right)=\theta_{\mathrm{i}}\left(\phi-\mathrm{T}-\mathrm{p}_{\mathrm{O} 2}\right)$ \\
\hline
\end{tabular}




\begin{tabular}{|c|c|c|}
\hline Membership fee & $\mathrm{U}_{\mathrm{S} 1}\left(\mathrm{p}_{\mathrm{S} 1}\right)=\theta_{\mathrm{i}} \phi-\mathrm{p}_{\mathrm{S} 1}$ & $\mathrm{U}_{\mathrm{S} 2}\left(\mathrm{p}_{\mathrm{S} 2}\right)=\theta_{\mathrm{i}} \phi-\mathrm{p}_{\mathrm{S} 2}$ \\
\hline
\end{tabular}

The competitive behavior between the two companies can be viewed as a two-stage game process: in the first stage, the companies choose the charging mode at the same time, and in the second stage, sets the product price.

The selection of the two companies in the first stage may have the following four situation combinations, and the existence conditions of equilibrium are discussed separately for each combination below.

Portfolio 1 One company chooses transaction fee and the other chooses membership fee.

Ignore product differences and company differences without loss of generality, so that company 1 chooses transaction fee and company 2 chooses membership fee. Record the price of the transaction fee $a s p_{O}$ and the price of the membership fee as $p_{S}$. According to the first-order condition, the optimal profits of the two companies are:

$$
\begin{gathered}
\Pi_{1}\left(p_{S}, p_{O}\right)=\frac{N \theta_{H}(\phi-T)}{8} \\
\Pi_{2}\left(p_{S}, p_{O}\right)=\frac{N \theta_{H} \phi}{4}
\end{gathered}
$$

If one company uses a transaction fee and the other company uses a membership fee, the company using transaction fee will choose the highest price that can be charged, and the company using membership fee will determine its own membership fee based on the competitors' price expectations. In this way, the only Nash equilibrium is obtained.

Similarly, we can get the equilibrium of several other portfolios.

Portfolio 2 Both companies choose transaction fee

The only equilibrium is that both companies provide products at marginal cost and can only make zero profits.

Portfolio 3 Both companies choose membership fee

The only equilibrium is that both companies provide products for free and can only make zero profits.

Portfolio 4 Both companies use two modes

As discussed in the two cases of Portfolio 2 and Portfolio 3, both the transaction fee method and the membership fee method will face the Bertrand price competition. The only equilibrium result is that the two companies provide free products, and they can only earn zero profits.

\section{Conclusion}

This article mainly analyzes how the platform chooses the charging mode under the consideration of psychological cost. The market structure of monopoly and duopoly are examined separately. The platform can choose to provide only transaction fee, only membership fee, or both fee models. In a monopolized market, if the platform only adopts one charging model, his choice mainly depends on the psychological cost and the relative size of the single-use utility. When the psychological cost is low, the monopolist will choose transaction fee; else it will choose membership fee. If a monopolist can use both modes, the common use of the two models is usually the preferred choice of the monopolist. In a duopoly market, the only pure strategy Nash equilibrium is that the two companies choose different pricing modes. Pure strategy Nash equilibrium and mixed strategy Nash equilibrium have always existed if two suppliers play a sequential game or play multiple games.

\section{Acknowledgements}

This paper is founded by Humanities and Social Sciences Research of the Ministry of Education Planning Fund "Research on Compatible Incentives of Differentiated Platforms and Market Structure Evolution (16YJA790024)”. 


\section{References}

[1] Rochet, J., Tirole. J. (2003) Platform competition in two-sided markets. Journal of European Economic Association. 4, 990-1029.

[2] Armstrong M. (2006) Competition in two-sided markets. RAND Journal of Economics, 3,668-691.

[3] Chen Peng, Xiong Li, Yang Lu. (2018) Research on the Impact of Charging Mode of Digital Content Platform on Consumer Behavior. Journal of Management Engineering, 3, 134-141. (in Chinese)

[4] Xia Dejian, Wang Yong. Research on Market Equilibrium of E-commerce Platform Based on the Difference of Logistics Aging Demand. Commercial Research, 2018(03): 171-176. (in Chinese)

[5] Liu Weiqi, Zhang Su. (2017) Analysis of Pricing Problems under the Bilateral Platform Merger Strategy. China Management Science, 5, 17-24. (in Chinese)

[6] Thaler R. (1985) Mental Accounting and Consumer Choice. Marketing Science, 3, 199- 214.

[7] Prelec D, Loewenstein G. (1998) The Red and the Black: Mental Accounting of Savings and Debt. Marketing Science, 1, 4-28.

[8] Soman D. (2001) The mental accounting of sunk time costs: Why time is not like money. Journal of Behavioral Decision Making, 3,169-185.

[9] Shy O. (2008) Measuring the cost of making payment decisions. Journal of Socio-Economics, 6, 2411-2416.

[10] Dutta R, Jarvenpaa S, Tomak K. (2013) Behavioral Economics of Digital Content, European Conference on Information Systems.

[11] Bi Wenjie, Chen Genyu, Chen Xiaohong. (2015) Research on Dynamic Pricing Problem Considering Consumer Dual Channel Psychological Account and Reference Dependence. China Management Science, 7, 142-151. (in Chinese)

[12] Li Aimei, Hao Mei, Li Li, Ling Wenyu. (2012) A New Perspective of Consumer Decision Analysis: Dual-channel Psychological Account Theory. Advances in Psychological Science, 11, 1709-1717. (in Chinese) 